\title{
ArcheoSciences
}

Revue d'archéométrie

$30 \mid 2006$

Varia

\section{Premières analyses métallographiques des produits sidérurgiques trouvés sur le site médiéval de Castel-Minier (Aulus-les-Bains, 09)}

First metallographic analyses of ferrous products found on the medieval site of Castel-Minier (Aulus-les-Bain, 09)

Philippe Dillmann, Florian Téreygeol et Catherine Verna

\section{OpenEdition}

Journals

Édition électronique

URL : https://journals.openedition.org/archeosciences/122

DOI : 10.4000/archeosciences. 122

ISBN : 978-2-7535-1595-6

ISSN : 2104-3728

Éditeur

Presses universitaires de Rennes

\section{Édition imprimée}

Date de publication : 31 décembre 2006

Pagination : 7-14

ISBN : 978-2-7535-0456-1

ISSN : 1960-1360

Référence électronique

Philippe Dillmann, Florian Téreygeol et Catherine Verna, « Premières analyses métallographiques des produits sidérurgiques trouvés sur le site médiéval de Castel-Minier (Aulus-les-Bains, 09) »,

ArcheoSciences [En ligne], 30 | 2006, mis en ligne le 31 décembre 2008, consulté le 01 février 2022.

URL : http://journals.openedition.org/archeosciences/122 ; DOI : https://doi.org/10.4000/

archeosciences.122 


\title{
Premières analyses métallographiques des produits sidérurgiques trouvés sur le site médiéval de Castel-Minier (Aulus-les-Bains, 09)
}

\author{
Philippe Dillmann*, Florian Tereygeol* et Catherine Verna**
}

\begin{abstract}
Résumé : Les découvertes récentes de deux ferriers contenant des produits et déchets de tous les stades de la chaîne opératoire sidérurgique sur le site médiéval de Castel-Minier, plus connu pour ses mines de plomb argentiferre, apporte un éclairage nouveau et fondamental sur la métallurgie et la diffusion des procédés dans cette région des Pyrénées. Cet article présente les premiers résultats métallographiques obtenus sur le métal mis au jour lors des sondages de ces ferriers au cours des fouilles 2004. Ils permettent de montrer que, très probablement, la production de cette installation sidérurgique est au moins en partie de l'acier dont la teneur en carbone est proche de l'eutectoïde. De plus, la composition des inclusions, indiquant une forte teneur en manganèse confirme que le minerai utilisé pour l'obtention du métal provient de la vallée voisine de Vicdessos, avec laquelle des échanges commerciaux sont attestés pour cette période par les sources historiques. Ces résultats ouvrent la voie à de futures études sur la sidérurgie utilisant des minerais au manganèse, caractéristique de cette région. En effet, le rôle de cet élément dans la production d'acier est encore mal compris bien que des minerais manganésiferres soient associés à ces types de production identifiés à l'époque médiévale entre autre dans les alpes italiennes et les Pyrénées.
\end{abstract}

\begin{abstract}
Recent excavations in the archaeological site of Castel-Minier, well known for its silver production, near the town of Aulus Les Bains (France), also reveal the presence of two iron slag heaps. This paper presents the first metallographical results on the iron artefacts found during the 2004 campaign. These artefacts are made of eutectoid steels and the slag inclusion analyses reveal the presence of high levels of manganese oxide. This indicates without doubt the use of a manganese ore coming from the neighbouring valley of Vicdessos. These first results are very important and lead the way for future studies on a specific ironmaking operating chain using manganese ore. Indeed, this element is found in the ore of two regions well known for producing steel in the medieval period (Italian Alps and Pyrenees) but its exact role in the mechanisms conducting to steel in a shaft furnace is not elucidated yet.
\end{abstract}

Mots clés : Fer, Manganèse, acier, Pyrénées.

Key words: Iron, manganese, steel, Pyrenees

\section{INTRODUCTION}

La zone pyrénéenne présente un intérêt particulier pour l'histoire de la sidérurgie au Moyen Âge. En effet, avec l'apparition de la mouline, forge de réduction directe profitant de la force hydraulique, elle témoigne de la large diffusion d'une innovation technique. Dès la fin du XIII ${ }^{e}$ siècle et au cours du XIV siècle, la mouline, qui correspond à une nou- velle façon de produire du fer dans un atelier où la force hydraulique est adaptée au fonctionnement du marteau et, au moins à partir du début du Xv siècle, à la soufflerie, entreprend la conquête des cours d'eau, de la Montagne Noire aux Pyrénées centrales et orientales.

À la lumière de ces données historiques et dans le cadre du programme ANR ACEUR et du programme de recherche archéologique sur le site de Castel-Minier (commune

* Chargé de Recherche CNRS, UMR 5060 Institut de Recherche sur les Archéomatériaux, Belfort et UMR 9956 Laboratoire Pierre Süe, CEA-CNRS, CEA, Saclay. (philippe.dillmann@cea.fr), (florian.tereygeol@cea.fr)

** Maître de conférence, Université Paris 12. 
d'Aulus-les-Bains, Ariège) ${ }^{1}$, la découverte de deux ferriers datés de cette période et contenant des déchets et produits de la chaîne opératoire complète (de la réduction du minerai à la forge de pièces en métal) s'avère de première importance. En effet, elle permet de réinterroger la complexité d'un site métallurgique plus connu pour l'exploitation médiévale de plomb argentifère que pour celle du fer. Elle offre en outre un nouvel éclairage à la question des échanges économiques noués entre la vallée du Garbet dans la région du Couserans où se situe Castel-Minier et les vallées environnantes. Enfin l'étude archéométrique des installations sidérurgiques de cette époque et de la compréhension de leur fonctionnement ainsi que du type de métal qu'elles produisent et un point crucial de ces études pluridiciplinaires.

À l'échelle du site de Castel Minier, il apparait manifeste que la métallurgie extractive du fer trouve a priori sa raison d'être dans la production d'argent. La présence d'une métallurgie du fer sur un site métallique, qu'il soit d'argent, de cuivre ou de plomb, n'est pas en soi étrange. Les exemples de forge associés à des mines sont nombreux $^{2}$. Pourtant, dans le cas présent, il ne s'agit pas d'une activité de transformation ou de mise en forme mais bien d'une production de fer métal à partir d'un minerai. Or, les données historiques et géologiques montrent qu'il n'existe pas de minerai exploitable dans cette vallée. En effet, il est certain que le ravitaillement en minerai des exploitations sidérurgiques de la vallée du Couserans et notamment des moulines quand elles sont attestées est absolument dépendant d'un bassin minier proche, puisqu'il se situe au-delà du port de Saleix : la vallée de Vicdessos. Cette grande vallée sidérurgique s'impose comme un centre majeur de production de fer et d'acier et un épicentre de l'espace technique circonscrit par l'innovation puisque son minerai est acheminé, sous haute surveillance, au-delà de la vallée et approvisionne de façon très contingentée et irrégulièrement les moulines des autres vallées du comté de Foix, du pays d'Olmes et parfois celles du Minervois. Ainsi, les conditions d'approvisionnement du Couserans sont-elles exceptionnelles, puisque seul cet espace dispose d'un apport régulier en minerai, reconnu, en outre, par un document officiel. Cette situation, qui paraît au premier abord privilégiée, doit être analysée à la lumière d'un rapport de force. La qualité des minerais de la vallée de Vicdessos explique que leur exportation soit sous le contrôle des notables de la communauté. Rappelons que, dès la fin du XIII ${ }^{e}$ siècle,

1. Programme P 25 du ministère de la Culture, Région Midi-Pyrénées, arrêté préfectoral $n^{\circ} 82 / 04$, fouille programmé, titulaire F. Téreygeol.

2. Benoît 1997, Bailly-Maitre 1994, Grandemange 1990. le minerai extrait de la mine de Sem (mont Rancié) est désigné sous la forme mineriis de ferro calybe vel axero ou " mine d'acier " et qu'il est utilisé, effectivement, pour la production d'acier. C'est en 1348 que la communauté, sous la pression du comte de Foix, accepte de le voir régulièrement exporté vers le Couserans. L'accord est signé entre le comte de Foix, le vicomte de Couserans et la communauté. Dans cet acte sont mentionnées les moulines alors implantées en Couserans. L'accord de 1348 peut être lu à la lumière des nouvelles conditions d'approvisionnement en bois que subit la sidérurgie de Vicdessos. Si la communauté consent à cette exportation, c'est contre la livraison du bois et du charbon de bois qui lui est absolument nécessaire. Cet échange est fondamental. Il demeurera en application jusqu'à la fin $\mathrm{du} \mathrm{XVIII}^{\mathrm{e}}$ siècle et se trouve à l'origine d'une déforestation accrue du Couserans dès le Moyen Âge et d'une préservation du couvert forestier de la grande vallée sidérurgique du Vicdessos, au moins jusqu'au XVII ${ }^{\mathrm{e}}$ siècle.

Au vu de ce bref aperçu des informations données par les sources historiques, on voit l'intérêt que peut présenter l'examen des produits de la forge de Castel Minier. Ceux-ci permettront de vérifier l'utilisation du minerai de Vicdessos dans la vallée du Couserans aux époques considérées. Rappelons que le minerai de la vallée de Vicdessos ${ }^{3}$ se caractérise par une teneur en manganèse importante (Verna, 2001). Cette teneur devrait se retrouver dans les inclusions non métalliques emprisonnées au sein du métal. Les inclusions, constituées de composés non réduits du minerai, portent, entre autre, la signature chimique du minerai (Dillmann et al., 2002; Dillmann et al., 1997). Si l'utilisation d'un minerai au manganèse est avérée, l'étude des produits et déchets de la chaîne opératoire correspondante sera de toute première importance. En effet, on connait historiquement le lien entre les minerais au manganèse et les filières produisant de l'acier, par exemple pour le Moyen Âge, dans les Pyrénées (Verna, 2000; Verna, 2001) ou dans les alpes italiennes (Braunstein, 2001). Or, le rôle de cet élément pour l'obtention de l'acier en chaîne opératoire directe est très mal connu. En effet, la thermodynamique montre que dans les conditions du bas-fourneau, les oxydes de cet élément ne sont pas réductibles. Le manganèse peut alors difficilement jouer le rôle carburigène (favorisant la formation de carbures) qui lui est connu en métallurgie contemporaine. Ce point reste donc à éclaircir car, il pourrait éventuellement y avoir formation de carbures de manganèse dans le métal lors de la réduction du minerai de fer manganésifere. Cependant, ici encore, on saisit mal le rôle de ces éventuels carbures de manganèse sur la formation d'acier.

3. Provenant d'un gisement identifié actuellement comme celui du Rancié. 
Cet article présente les premiers résultats métallographiques obtenus sur les produits de la chaîne opératoire trouvée sur le site de Castel minier. Ces résultats seront mis en perspective des différents points cités précédemment et serviront de base à une étude complète lors de la poursuite des fouilles dans les prochaines années.

\section{LES FERRIERS DE CASTEL-MINIER : MISE AU JOUR DE PRODUITS METALLIQUES}

Le but n'est pas ici de détailler les sondages effectués dans ces ferriers. Pour plus d'information on pourra se reporter à l'article de Bonnamour et al. (2007) dans le même volume. Les prospections menées à Castel-Minier en 2003 et 2004 ont permis de localiser deux ferriers ${ }^{4}$. Le premier sondage archéologique entrepris sur le ferrier le plus important donne une stratigraphie relativement simple. Passée la couche de terre végétale, on note sur près d'un mètre d'épaisseur une accumulation de scorie dont la monotonie n'est rompue que par un lit argilo-sableux ocre. En revanche, il est très clair que le dépôt de ces scories est soigné. Il est limité à l'ouest par un muret qui empêche les couches de s'épandre dans le chenal qui est encore actif aujourd'hui. Ce muret est bien bâti et ne peut être assimilé à un simple mur terrasse. Sa disposition dans le sens de la pente l'interdit. La faible emprise du sondage ne permet pas de comprendre l'ensemble de ce dépôt. Faute de céramique, une datation par ${ }^{14} \mathrm{C}$ a été réalisée donnant les XIII ${ }^{\mathrm{e}}$-XIV ${ }^{\mathrm{e}}$ siècle comme la fourchette chronologique la plus probable ${ }^{5}$. Il ne s'agit bien sûr que d'une seule date ${ }^{14} \mathrm{C}$ mais elle concerne des charbons pris dans la strate supérieure du ferrier. On supposera donc en bonne logique que la strate inférieure est plus ancienne. L'activité sidérurgique est donc ancienne sur le site. Elle est en tout cas contemporaine de l'activité minière dont une phase $\mathrm{XIII}^{\mathrm{e}}$ siècle a été caractérisée en mine. D'autre part, dans ce sondage de faible ampleur, il a été possible de mettre au jour en plus des scories et des parois de fours, un morceau de plaque et une masse métallique parallélépipédique que nous nommerons dans la suite " $\operatorname{lingot}^{6}{ }$. Ce sont donc

4. Bonnamour et al., 2005.

5. Datation ARC 2438, Âge ${ }^{14} \mathrm{C}$ conventionnel : $715+/-45 \mathrm{BP}$ (sigma 13C estimé de $-25,00$ pour mille vs PDB) Date $14 \mathrm{C}$ calibrée : $1220 \mathrm{cal}$ AD - 1390 cal AD (courbe de calibration "IntCal98 ", Stuiver et al., 1998, Radiocarbon, 40).

6. Le terme de lingot est à prendre avec prudence. Il s'agit ici d'un métal déjà compacté, par opposition à la loupe, et non encore mis en forme et ne pouvant être considéré comme un produit semi fini. Pour plus de détail à ce sujet on pourra se référer au manuel de Mangin, M., 2004. Le Fer, Paris, Errance, 239 p. des éléments provenant de différents stades de la chaîne opératoire que nous avons exhumés. Leur analyse constitue le premier pas d'approche analytique globale sur le thème de la production du fer à la période charnière des $\mathrm{XIII}^{\mathrm{e}}$ et XIV ${ }^{\mathrm{e}}$ siècle dans les Pyrénées.

Le « lingot » se présente sous la forme d'un bloc parallélépipédique pesant environ $800 \mathrm{~g}$ et dont les dimensions sont d'environ $9 \times 5 \times 3 \mathrm{~cm}$ (Fig. 1). Une des faces semble perturbée et la forme retrouvée pourrait avoir appartenu à un objet de taille plus importante (barre?) dont une extrémité aurait été tranchée à chaud.

La plaque présente une forme plate avec des dimensions d'environ 8 x 4 x $1 \mathrm{~cm}$ pour une masse d'environ $130 \mathrm{~g}$. Elle est légèrement plus épaisse à une de ses extrémités. Il semble, là encore que ce soit un fragment d'une pièce plus importante.

\section{Méthodes analytiques}

Après découpe, les échantillons ont été dégrossis aux papiers abrasifs SiC grades 80 à 1200 . Un polissage de finition a été effectué à l'aide de suspensions diamantées Struers 9,3 et $6 \mu \mathrm{m}$. La structure métallographique du métal a été observée par microcopie optique après attaque au Nital $2 \%$ (Pokorny et Pokorny, 1994).

Les analyses de la composition des inclusions ont été effectuées au Microscope Électronique à Balayage (MEB)
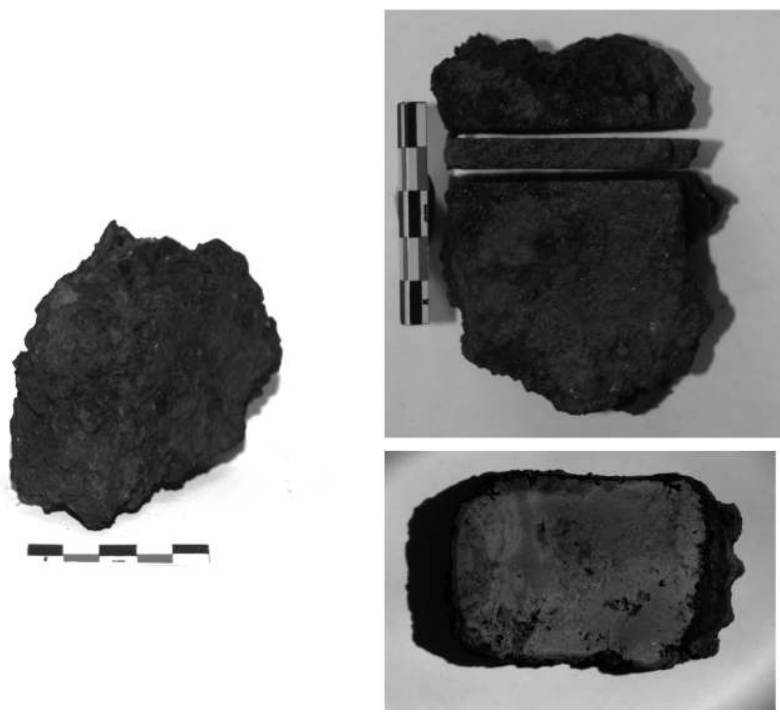

Figure $1:$ «Lingot » trouvé sur le site de Castel-Minier. Prélèvement de l'échantillon et vue de la face polie après attaque Nital. Figure 1: "Ingot" found on the Castel-Minier site. Sampling-Polished cross-section after Nital etching. 


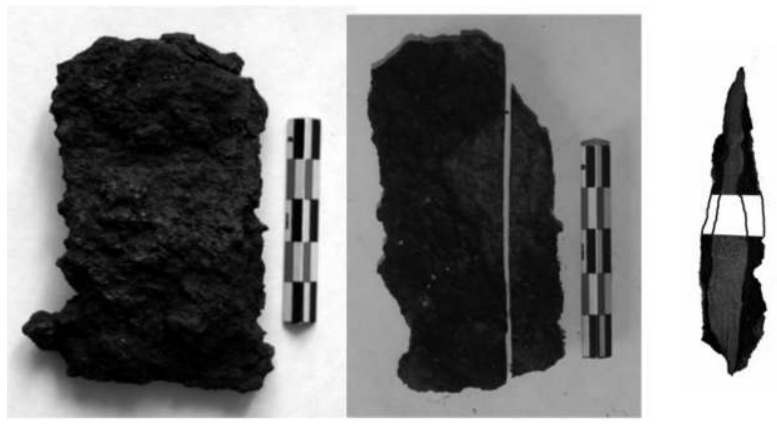

Figure 2: "Plaque» trouvée sur le site de Castel-Minier. Prélèvement de l'échantillon. Vue de la face polie.

Figure 2: "Plate" found on the Castel-Minier site. Sampling and polished cross-section.

par Spectrométrie Dispersive en Énergie (EDS) avant toute attaque métallographique ou après repolissage. Le détecteur $\mathrm{Si}(\mathrm{Li})$ utilisé est équipé d'une fenêtre mince qui permet la quantification de l'oxygène avec une bonne précision (1\% sur un échantillon standard de magnétite - Tension d'accélération des électrons : $15 \mathrm{kV}$ ). La limite de détection est de l'ordre de $0,5 \%$ mass pour la détection des éléments de $\mathrm{n}^{\circ}$ atomique supérieur au magnésium et l'incertitude relative de $1 \%$ (sur références NIST de verres et oxydes de fer). Comme précisé dans de précédentes publications (Dillmann et Balasubramaniam, 2001; Dillmann et al., 2003; Dillmann et al., 2003 paru en 2004; L'Héritier, 2004; L'Héritier et al., 2004; L'Héritier, 2002), une étude fiable des inclusions ne peut se faire que si au moins une cinquantaine d'inclusions sont analysées par objets. Cette démarche permet de minimiser les effets de fragmentation ${ }^{7}$ observables sur certaines inclusions et dus au travail d'épuration. Les teneurs sont quantifiées en pourcentages massiques et convertis en oxydes.

Afin de détecter la présence éventuelle de manganèse dans le métal, la micro Fluorescence $X(\mu \mathrm{XRF})$ sur un générateur de rayons $\mathrm{X}$ à anode tournante dont le faisceau est concentré à l'aide d'un capillaire de borosillicate (Engstrom et Riekel, 1996) sur une surface de $20 \times 20 \mu \mathrm{m}^{2}$ a été pratiquée sur la plaque. Les rayons $\mathrm{X}$ sont produits par une anode de Molybdène. Les spectres de fluorescence X ont été collectés à l'aide d'un détecteur Germanium pour des durées de $30 \mathrm{mn}$ par point analysé. Le manganèse a été recherché en analysant le métal et en évitant toute inclusion. Avec l'appareillage utilisé, la limite de quantification de cet élément dans une matrice de fer est d'environ 70 ppm.

7. Cette fragmentation provoque un enrichissement en certains éléments donnant pour quelques inclusions de l'objet des compositions parfois fort éloignées de la majorité des inclusions et souvent liées à la présence d'un seul minéral dans cette inclusion (fayalite, hercynite, etc.).

\section{Résultats}

\subsection{Structure et composition du métal}

La face polie provenant du « lingot " présente un grand nombre de porosités, dont certaines sont millimétriques. Dans certains cas, celles-ci sont remplies d'oxydes et d'oxyhydroxydes de fer, générés par la corrosion lors de l'enfouissement de l'objet. Certaines de ces porosités sont partiellement remplies par des inclusions non métalliques, résidus de scories de la phase de réduction. En d'autres endroits, seules ces inclusions scoritiques sont identifiées (absence de porosité). Elles sont constituées de phases amorphes (Fig. 3). Vu le nombre de porosités et d'inclusions assez important, cet objet métallique se situe dans la chaîne opératoire proche de la phase de réduction tout en étant, vu sa forme extérieure, déjà travaillé, épuré et compacté de manière importante. Les observations au microscope optique après attaque au réactif nital montrent que la totalité de la surface est constituée d'acier très carburé, presque entièrement eutectoïde $\left(0,8 \%_{\text {mass }}\right.$ de carbone) avec des écarts de part et d'autre de cet eutectoïde allant jusqu'à $0,1 \%{ }_{\text {mass }}$.



Figure 3: "Lingot» (gauche) inclusion amorphe dans matrice perlitique, (droite) structure eutectoïde perlitique.

Figure 3: "Ingot" (left) amorphous inclusion in a pearlitic matrix (right) eutectoïd steel structure.

Les analyses métallographiques effectuées sur la plaque révèlent que l'objet est entièrement constitué d'un acier eutectoïde $\left(0,8 \%{ }_{\text {mass }} \mathrm{C}\right)$ avec une très légère baisse de cette teneur vers la partie la plus épaisse de l'objet $\left(0,7 \%_{\text {mass }} \mathrm{C}\right.$ Figure 4). La perlite présente une structure assez fine, ce qui dénote un refroidissement relativement rapide. Un grand nombre d'inclusions a été observé dans la structure métallique : la propreté inclusionnaire est homogène et relativement mauvaise sur la totalité de la pièce. Ces inclusions sont allongées dans le sens perpendiculaire au martelage. L'ensemble des inclusions analysées présente un aspect amorphe (Fig. 4). Contrairement au " lingot ", cet objet ne présente aucune porosité. Ceci est vraisemblablement dû au fait 


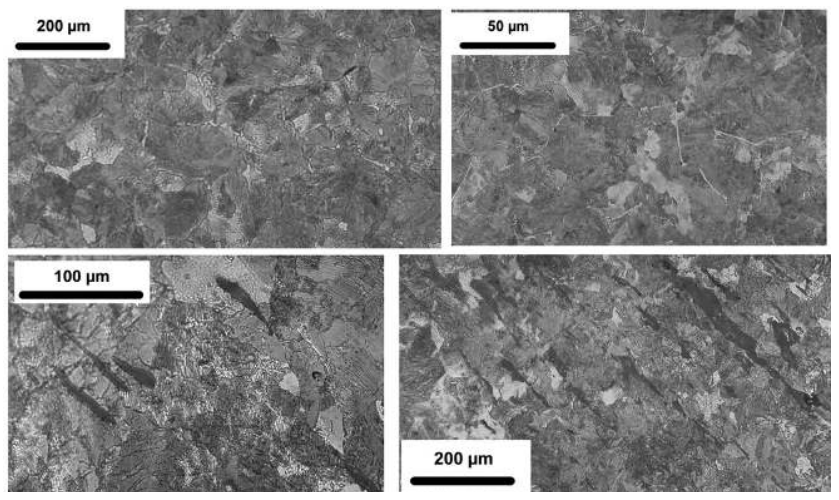

Figure 4 : Plaque (haut) structure ferrito-perlitique à $0,7 \%$ de carbone à perlitique ( $0,8 \%$ de carbone) ; (bas) inclusions amorphes dans structure aciérée perlitique.

Figure 4: Plate (up) ferrito-pearlitic structure (0.7\%) to pearlitic (0.8\%) (down) amorphous inclusion in a pearlitic steel structure.

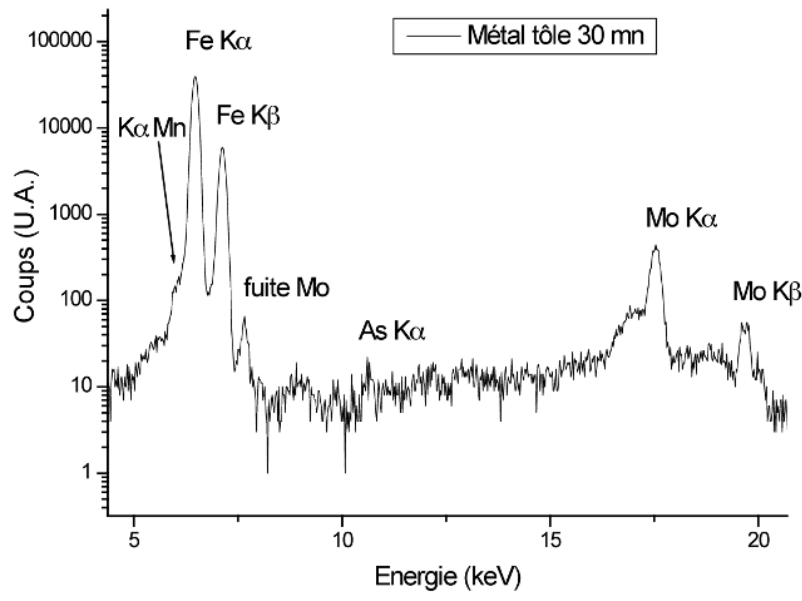

Figure 5 : Spectre de $\mu$ XRF (taille de faisceau $20 \times 20 \mu \mathrm{m}^{2}$ ) caractéristique obtenu sur le métal de la plaque.

Figure 5: $\mu X R F$ spectrum (beamsize $20 \times 20 \mu \mathrm{m}^{2}$ ) obtained in a metallic zone.

qu'il provient d'une étape postérieure de la chaîne opératoire, et a vu un martelage plus poussé que le lingot pour sa mise en forme ${ }^{8}$. Des analyses de composition du métal dans les zones exemptes d'inclusions ont été réalisées par $\mu \mathrm{XRF}$. Sur les spectres obtenus, un léger épaulement à gauche de la raie $\mathrm{K}_{\alpha} \mathrm{du}$ fer correspond à la raie $\mathrm{K}_{\alpha}$ du manganèse. En tout état de cause, ces teneurs sont inférieures à $60 \mathrm{ppm}$. Au sens métallurgique, il semble que le métal de la plaque ne contient pas de manganèse. Ces analyses seront cependant à poursuivre plus en détail dans de futures études.

8. On trouvera plus de détails sur les différentes étapes de la chaîne opératoire et sur les types de martelage et durées qu'elles impliquent dans l'article de L'Héritier et al. (2006).

\subsection{Analyse des inclusions}

Les analyses EDS effectuées sur les inclusions révèlent la présence des éléments majeurs oxygène, fer, silicium, aluminium, calcium, potassium, magnésium et manganèse. La teneur en oxyde de fer (exprimé en $\mathrm{FeO}$ ) varie fortement dans les inclusions. Ceci peut s'expliquer par le fait que, en fonction des conditions locales de température et de pression dans le bas-fourneau, la réduction de ces oxydes sera plus ou moins poussée. La figure 6 montre l'évolution de la teneur en $\mathrm{FeO}$ dans les inclusions en fonction de la teneur en $\mathrm{SiO}_{2}$, composé non réduit dans les conditions du bas fourneau. On voit que la teneur en $\mathrm{FeO}$ diminue en fonction de l'augmentation de celle en $\mathrm{SiO}_{2}$ dans les inclusions. Un point important est a noter : l'existence d'inclusions à très faible teneur en $\mathrm{FeO}\left(<5 \%{ }_{\text {mass }}\right)$. Celles-ci semblent correspondre à des zones où les conditions dans le bas fourneau sont particulièrement réductrices pour l'oxyde de fer ${ }^{9}$. Les teneurs en $\mathrm{MnO}$ des inclusions sont très importantes $\left(4<\%{ }_{\text {mass }} \mathrm{MnO}\right.$ $<25)$. Cet élément ne peut provenir que du minerai initial et de telles teneurs sont sans aucun doute à mettre en relation avec l'utilisation d'un minerai au manganèse. On constate également sur la figure 6 que le comportement du $\mathrm{MnO}$ est différent de celui du $\mathrm{FeO}$ et ne semble pas décroître en fonction de l'augmentation de la teneur en $\mathrm{SiO}_{2}$. De plus, le rapport $\mathrm{MnO} / \mathrm{SiO}_{2}$ n'est pas constant dans les inclusions, ce qui aurait indiqué sans aucun doute que cet oxyde n'est pas réduit dans le bas-fourneau. Ce point restera à être éclairci dans la suite des études.

Les autres oxydes présents dans les inclusions ne sont pas réduits dans le bas fourneau. Ils peuvent provenir soit du minerai soit d'une pollution pendant la réduction par les parois du fourneau ou le charbon de bois. Ils peuvent également provenir d'éventuels ajouts. Toujours est il que, s'ils ne sont pas réduits, leur rapport respectif ne doit pas changer pour toutes les scories produites par une même réduction ${ }^{10}$. $\mathrm{Si}$ ceci est vrai pour les scories macroscopiques, cela n'est pas toujours le cas pour les inclusions, de taille microscopiques et pouvant subir des fragmentations. Cependant, la figure 7 montre que c'est le cas pour les deux objets analysés pour lesquels les rapports $\mathrm{Al}_{2} \mathrm{O}_{3} / \mathrm{SiO}_{2}$ et $\mathrm{CaO} / \mathrm{K}_{2} \mathrm{O}$ sont relativement constants pour l'ensemble des inclusions de l'objet. Certaines inclusions ne semblent pas respecter ce rapport,

9. Certaines inclusions à forte teneur en $\mathrm{SiO}_{2}$ et faible teneur en $\mathrm{FeO}$ peuvent correspondre à des ajouts sableux lors des soudures. Mais ce n'est pas le cas ici car ces inclusions contiennent presque toutes une forte proportion de manganèse. De plus, pour ces inclusions, le rapport $\mathrm{Al}_{2} \mathrm{O}_{3} / \mathrm{SiO}_{2}$ est identique à celui observé sur les autres inclusions (voir plus bas). Elles proviennent donc bien de composés non réduits du minerai.

10. Si celles-ci passent à l'état liquide pendant la réduction, ce qui est la plupart du temps le cas. 

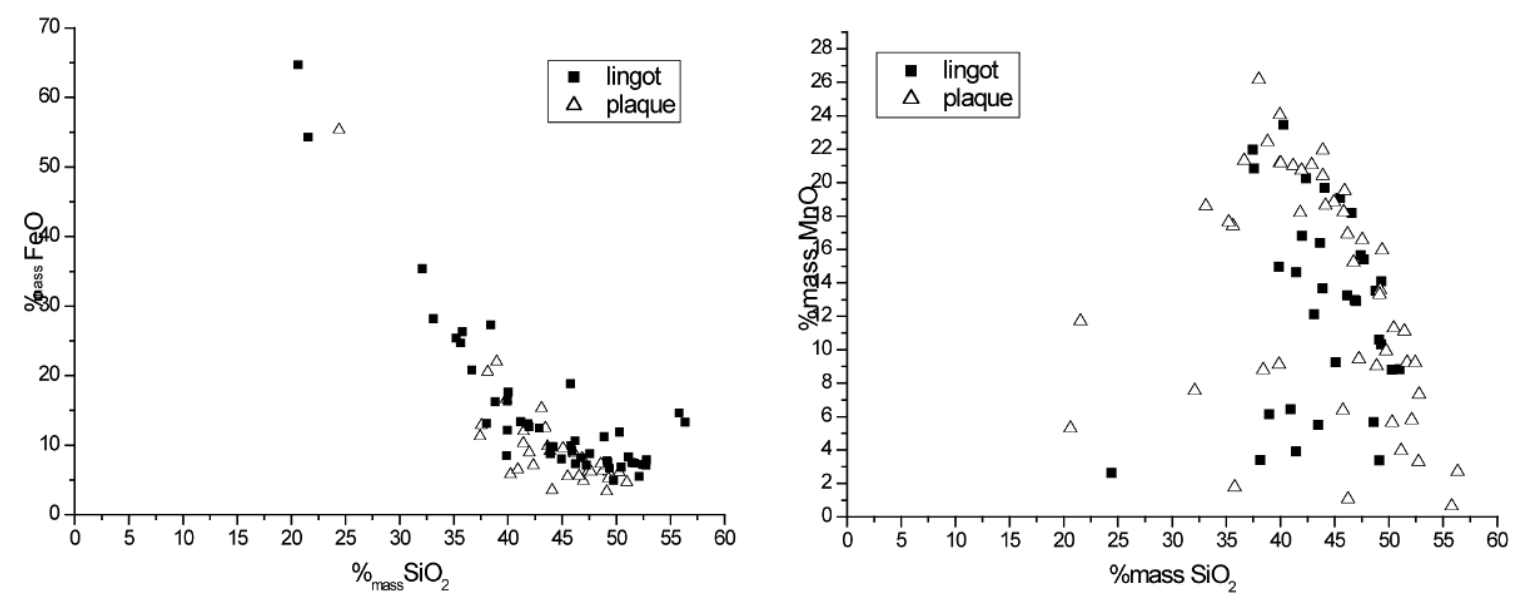

Figure 6 : (gauche) teneurs en $\mathrm{FeO}$ en fonction de $\mathrm{SiO}_{2}$ dans les inclusions du « lingot » et de la plaque. (droite) teneurs en $\mathrm{MnO}$ en fonction de $\mathrm{SiO}_{2}$ dans les inclusions du « lingot " et de la plaque.

Figure 6: (left) $\mathrm{FeO}$ content versus $\mathrm{SiO}_{2}$ in slag inclusion from "ingot" and plate (right) $\mathrm{MnO}$ content versus $\mathrm{SiO}_{2}$.
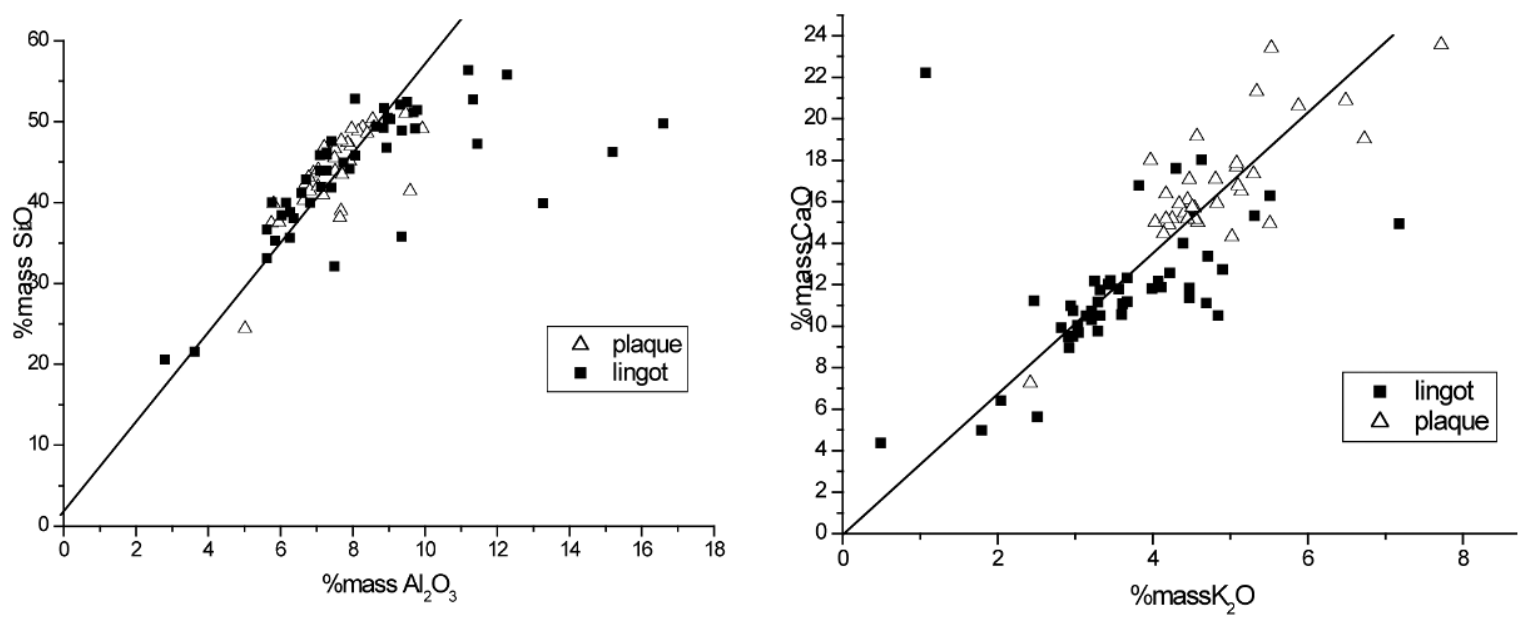

Figure 7 : (gauche) teneurs en $\mathrm{Al}_{2} \mathrm{O}_{3}$ et $\mathrm{SiO}_{2}$ dans les inclusions du « lingot » et de la plaque. (droite) teneurs en $\mathrm{K}_{2} \mathrm{O}$ et $\mathrm{CAO}$ dans les inclusions du «lingot » et de la plaque.

Figure 7: (left) $\mathrm{Al}_{2} \mathrm{O}_{3}$ and $\mathrm{SiO}_{2}$ in slag inclusions from "ingot" and plate (right) $\mathrm{K}_{2} \mathrm{O}$ and $\mathrm{CAO}$ contents.

elles peuvent soient correspondre à des ajouts lors du forgeage soit, plus vraisemblablement êtres plus fragmentées que les autres. Il semble cependant que les rapports identifiés sur la majorité des inclusions puissent «signer » les réductions effectuées sur le site de Castel-Minier. Celui-ci sera comparé à ceux mesurés sur les scories macroscopiques dans la suite des études.

\section{Discussion}

Ces premières analyses métallographiques appellent un certain nombre de commentaires en relation avec les questions évoquées dans l'introduction. En premier lieu il convient de rappeler le faible nombre d'objets examinés. L'ensemble de ces observations devra donc être confirmé par l'étude des déchets (scories) et autres produits qui seront mis au jour lors des fouilles des années à venir. 
Il semble cependant que la production de la forge d'Aulus soit au moins en partie de l'acier très carburé. Dans tous les cas les teneurs observées sont de l'ordre de l'eutectoïde. Ces teneurs importantes en carbone semblent donc confirmer les données historiques. On peut dès lors se poser la question sur la forme des produits qui sortaient de cette forge (barres ou plaques). Enfin, aucun traitement thermique destiné à durcir l'acier n'a pu être mis en évidence sur les objets, l'ensemble des structures observées correspondant à des refroidissements beaucoup plus lents que ceux nécessaires à une trempe.

La très forte teneur en manganèse observée dans les inclusions va dans le sens de l'utilisation du minerai en provenance de la Vallée de Vicdessos. Les études sur la composition chimique de ce minerai (éléments majeur et présents à l'état de traces) seront menées à bien dans les mois à venir mais a priori aucun autre minerai manganésifere n'est accessible dans la vallée du Couserans.

L'utilisation d'un minerai au manganèse, si elle est assurée, pose un certain nombre de questions. Au vu des teneurs très faibles mesurées dans la matrice métallique et de celles très importantes mesurées dans les inclusions, il semble que cet élément ne soit pas réduit et ne passe pas dans le métal (ou tout du moins en quantité trop faible pour avoir une influence métallurgique). La dispersion de ces teneurs et notamment la variabilité observée pour le rapport $\mathrm{MnO} /$ $\mathrm{SiO}_{2}$ (ce qui n'est pas le cas pour les rapports des composés non réduits dans les inclusions des deux objets) reste cependant encore à être comprise.

\section{Conclusion}

Les premiers résultats métallographiques présentés ici montrent le potentiel du site de Castel-Minier pour une étude d'une chaîne opératoire sidérurgique qui semble typique de la région à cette époque. Les observations archéométriques vont dans le sens des éléments historiques et techniques déjà connus : production au moins en partie d'acier fortement carburé, utilisation d'un minerai au manganèse, qui devront dans le futur être mis en perspective d'une étude plus large portant à la fois sur l'ensemble des produits et déchets mis au jour sur le site et sur les résultats archéologiques et archéométriques obtenus sur d'autres sites de la région à des périodes contemporaines ou postérieures. Un second ferrier n'a pas fait l'objet d'investigation intrusive mais les données topographiques de ce dernier permettent d'avancer l'hypothèse de l'existence d'une forge rattachée à la fortification de Castel-Minier. Parallèlement, des objets en fer ont été exhumés à l'occasion d'autres sondages sur le castrum. S'il est bien certain que la raison d'être de ce site repose sur l'exploitation de l'argent, nous pouvons avoir la chance de disposer de la totalité de la chaîne opératoire de production du fer en un même lieu. La fouille de ces amas de scories et la recherche des structures métallurgiques qui leur sont liées permettront de compléter avantageusement le tableau de cette activité sidérurgique médiévale d'autant plus qu'un usage de la force hydraulique dans ce système de production ne peut être exclu.

\section{Bibliographie}

Bonnamour, G., Florsch, N., Tereygeol, F., 2007. Les prospections des ferriers de Castel-Minier, Revue d'Archéométrie, 30, p. xx.

Braunstein, P., 2001. La sidérurgie alpine en Italie (XII-XVII siècle), Rome, École Française de Rome, p. 290.

Dillmann, P., Balasubramaniam, R., 2001. Characterization of ancient Indian iron, entrapped slag inclusions using electron, photon and nuclear microprobes, Bull. Mater. Sci., 24, (3), p. 317-322.

Dillmann, P., Bernardi, P., Fluzin, P., 2003. Use of iron for the building of medieval monuments. The Palais des Papes in Avignon and other french buildings, Archaeometallurgy in Europe, AIM, Milan, p. 199-208.

Dillmann, P., Bernardi, P., Fluzin, P., 2004. Iron in medieval monuments. Metallographic analysis of irons coming from the Palais des Papes in Avignon, Revue d'Archéométrie, 27, p. 123-134.

Dillmann, P., Fluzin, P., Chevallier, P., 2002. Determination of ironmaking processes using synchrotron microprobe, British Archaeological Reports International Series, 1043, (II), p. $327-$ 344.

Dillmann, P. Populus, P., Chevallier, P., Fluzin, P., Béranger, G., Firsov, A., 1997. Microdiffraction Coupled with X ray fluorescence Microprobe. Application in Archaeometry, Journal of trace and microprobe techniques, 15, (3), p. 251-262.

ENGSTROM, P., RieKel, C., 1996. Low-angle synchrotron radiation diffraction with glass-capillary optics, Journal of Synchrotron Radiation, 3, p. 97-100.

L'Héritier, M., Juhin, A., Dillmann, P., Aranda, R., Benoit, P., 2006. Utilisation des alliages ferreux dans la construction monumentale du Moyen Âge. État des lieux de l'avancée des études métallographiques et archéométriques, Revue d'Archeométrie, 29, p. 117-126.

L'Héritier, M., 2004. L'utilisation du fer à la cathédrale de Rouen à l'époque médiévale, in J.-P. Watte (éd.), Haute-Normandie Archéologique, Eu, CRAHN, p. 69-78. 
L'Heritier, M., Dillmann, P., Benoît, P., 2004. Analyse métallographique de fers à cheval médiévaux. Mise en forme et origine du métal, La Revue d'Archéométrie, 27, p. 193-206.

L'Heritier, M., 2002. Approches typologique et archéométallurgique du fer à cheval médiéval, Paris, Université Paris I.

Mangin, M., 2004. Le Fer, Paris, Errance, 239 p.

Pокоrny, J., Рокоrny, A., 1994. Métallographie. Techniques de l'Ingénieur, M 90, volume MB, p. 1-52.
Verna, C., 2000. De la forge au marché des fers: les espaces emboîtés de la réduction directe, Gè curs d'arqueologia d'Andorra 2000, L'obtencio del ferro pel procediment directe entre els segles IV i XIX, Andorra, p. 63-78.

Verna, C., 2001. Le temps des Moulines. Fer, technique et société dans les Pyrénées centrales (XIII $-X V T^{e}$ siècles), Paris, Thèse de doctorat, Publications de la Sorbonne. 